\title{
Editorial: PRB's 50th Anniversary 1970-2020
}

Happy New Year! The old year of 2020 turned out to be very difficult because of the pandemic, but it will be a year to remember for the world's resilience and dedication to endure and prevail under extremely adverse circumstances. In the fiftieth year of the existence of Physical Review B, it was our editorial goal to stay the course and provide a sense of normality.

To mark our 50th anniversary, Physics Magazine published an article illuminating the original ideas behind three seminal PRB papers and their impact on present-day early career scientists [1]. The PRB editors also built a collection [2] of 50 "Milestone" papers from the last five decades that have made, in the opinion of the editors, lasting contributions to condensed matter and materials physics. PRB has published more than 190,000 papers since its inception, so many great papers had to be omitted. The editors strived to represent the many diverse subfields that we publish.

And as we look to the next 50 years we understand that researchers increasingly need to publish their research in an open-access (OA) venue. To meet this need, PRB offers authors the option of paying an article-processing charge (APC) to make a paper immediately accessible on publication under a CC BY 4.0 International license, the most accommodating license offered. Alternatively, authors needing to publish in a fully OA journal may consider submitting their manuscript to Physical Review Research, which was launched in 2019 and publishes papers in all areas of physics, including condensed matter and materials physics. The PRB editors contribute their expertise to evaluating papers submitted to PRResearch to ensure a similar editorial standard.

Another option of interest for our PRB authors is the new Letter article type: a short, significant, and readable paper for dissemination of important research results, and the established and recognized descriptor for these types of papers. This section replaces the Rapid Communications section of PRB, and helps authors by ensuring that researchers, funding agencies, and promotion and hiring committees clearly understand the impact and significance of the Letters published in PRB [3]. Letters are intended to be easy to digest. We therefore ask authors to adhere to the length limit (now 4500 words) and to carefully craft the introduction and main text to be understandable to PRB's broad readership of condensed matter and materials physicists. We also take this opportunity to remind authors that we encourage follow-up or joint submission of a PRL with a PRB Regular Article [4].

As an APS journal, PRB is managed by scientists for scientists, and our editors draw heavily on their organic connection with researchers [5]. As always, we value high-quality peer review of the papers we receive, essential to maintaining a high signal-to-noise ratio for trustworthy research results. The process relies on constructive, yet critical scrutiny by peers. Our community, with its distinct flavors of authors, readers, or referees, is the key to overall success, enabling the PRB editors, and the Editorial Board, to be impartial arbiters of each paper that we receive. With your help, we look forward to another 50 years 
of providing outstanding depth and breadth of coverage of research worldwide. We thank you and look forward to being able to meet you in person again.

Yonko T. Millev

Anthony M. Begley

Laurens W. Molenkamp

(d) Published 27 January 2021

DOI: 10.1103/PhysRevB.103.030001

[1] 50 Years of Physical Review B: Solid Hits in Condensed Matter Research, Physics 13, 29 (2020).

[2] PRB Milestone Collection: https://journals.aps.org/prb/50th.

[3] Announcement: Rapid Communications papers will now be Letters, https://journals.aps.org/edannounce/rapid-communications-will-beletters.

[4] Pierre Meystre, Editorial: Supplemental Material or Joint Submission? Phys. Rev. Lett. 118, 240001 (2017).

[5] Laurens W. Molenkamp, Editorial: Scope and Standards of PRB, Phys. Rev. B 87, 190001 (2013). 